\title{
Optical coherence tomography-based topography determination of corneal grafts in eye bank cultivation
}

\author{
Angela Damian \\ Berthold Seitz \\ Achim Langenbucher \\ Timo Eppig
}




\title{
Optical coherence tomography-based topography determination of corneal grafts in eye bank cultivation
}

\author{
Angela Damian, ${ }^{\mathrm{a}}$ Berthold Seitz, ${ }^{\mathrm{b}}$ Achim Langenbucher, ${ }^{\mathrm{a}}$ and Timo Eppig ${ }^{\mathrm{a}, *}$ \\ asaarland University, Institute of Experimental Ophthalmology, Kirrberger Street 100, Building 22, 66421 Homburg/Saar, Germany \\ ${ }^{b}$ Saarland University Medical Center (UKS), Department of Ophthalmology, Kirrberger Street 100, Building 22, 66421 Homburg/Saar, Germany
}

\begin{abstract}
Vision loss due to corneal injuries or diseases can be treated by transplantation of human corneal grafts (keratoplasty). However, quality assurance in retrieving and cultivating the tissue transplants is confined to visual and microbiological testing. To identify previous refractive surgery or morphological alterations, an automatic, noncontact, sterile screening procedure is required. Twenty-three corneal grafts have been measured in organ culture with a clinical spectral-domain optical coherence tomographer. Employing a biconic surface fit with 10 degrees of freedom, the radii of curvature and conic constants could be estimated for the anterior and posterior corneal surfaces. Thereupon, central corneal thickness, refractive values, and astigmatism have been calculated. Clinical investigations are required to elaborate specific donor-host matching in the future. (C) The Authors. Published by SPIE under a Creative Commons Attribution 3.0 Unported License. Distribution or reproduction of this work in whole or in part requires full attribution of the original publication, including its DOI. [DOI: 10.1117/1.JBO.22.1.016001]
\end{abstract}

Keywords: optical coherence tomography; keratoplasty; eye banking; corneal topography; donor cornea qualifying. Paper 160601R received Sep. 1, 2016; accepted for publication Dec. 5, 2016; published online Jan. 2, 2017.

\section{Introduction}

The transplantation of human corneal grafts (keratoplasty) has been performed for more than 100 years. ${ }^{1}$ In 2012, more than 184,500 corneal transplantations were carried out worldwide, about $90 \%$ of them were conducted as penetrating keratoplasty. ${ }^{2}$ About 3,628 keratoplasties were performed in Germany in $2012,{ }^{3}$ while 2 years later, the number increased by more than $57 \%(5,725){ }^{4}$ Thus, keratoplasty is recognized as the oldest, most frequently performed, and most successful transplantation of human tissue with a success rate of $\sim 90 \% .5,6$ Retrieval, cultivation, and storage of human ocular tissue are performed by eye banks, which are also in charge of the quality assurance and provision of the corneal grafts for keratoplasty. The donor cornea is usually harvested as a corneoscleral button. After excision, it is transferred to the organ culture, where it is preserved in a supplemented tissue culture medium that contains growth factors, antibiotics, antimycotics, and buffer components. This preservation maintains the corneal metabolism and causes folding of the posterior surface [Fig. 1(a)], stromal swelling, and epithelial shedding. ${ }^{78} \mathrm{~A}$ few days before transplantation, the swelling is reversed by re-immersing the cornea into a second culture medium additionally enriched with dextran as a dehydrating agent [Fig. 1(a)].

Being part of the human tissue and organ transplant system, the corneal graft is subject to a sophisticated inspection procedure to assure transplantation eligibility. ${ }^{9-12}$ The frequency of discarded grafts due to contamination, insufficient endothelium, and other reasons is about $30 \%$ as stated by Reinshagen et al. ${ }^{3,4}$ Donor cornea examination is usually performed with a slit lamp biomicroscope and a specular microscope. Assessments include

*Address all correspondence to: Timo Eppig, E-mail: timo.eppig@uks.eu visual inspection for alterations in tissue homogeneity as well as the determination of the endothelial cell density. In addition, donor liquor and swabs undergo microbiological examination to exclude contamination of the corneal button. Pathological changes, such as opacification, scars, or inflammations, can be identified by these techniques. However, manifestations of previous intraocular or anterior segment surgery, such as refractive corneal procedures (arcuate cuts or intrastromal refractive surgery), laser photoablation surgery [e.g., laser in-situ keratomileusis (LASIK) and photorefractive keratectomy (PRK)], or morphological alterations such as pterygia, superficial scars, (subclinical) keratoconus, or corneal dystrophies, may pass visual inspection unnoticed. ${ }^{13-15}$ Such pathological grafts may cause complications during surgery and moreover impair the achievable visual outcome. ${ }^{16,17}$ Supplementary screening methods are required which are able to visualize the structure of the corneal tissue and to calculate the surface topography or tomography. An objective, semiautomated screening method may assist standardization. An increasing number of potential donors have had refractive surgery and, therefore, the corneas may be unsuitable for penetrating keratoplasty, but not for endothelial lamellar keratoplasty. ${ }^{18}$ This requires an increase in the sensitivity and specificity of screening methods.

While identifying donor corneas potentially being unsuitable for transplantation as part of the eye banks' quality assurance procedure, the corneal grafts should stay within the sterile culture flask [Fig. 2(a)] filled with a culture medium for maintaining a sterile environment throughout any quality assessment procedure. Ultrasound biomicroscopy requires contact of the ultrasound probe with the measurement object, either with direct or with immersion fluid, and, therefore, appears unsuitable. Optical measurement techniques, however, are contact-free and can offer a better spatial resolution. The surfaces of the 

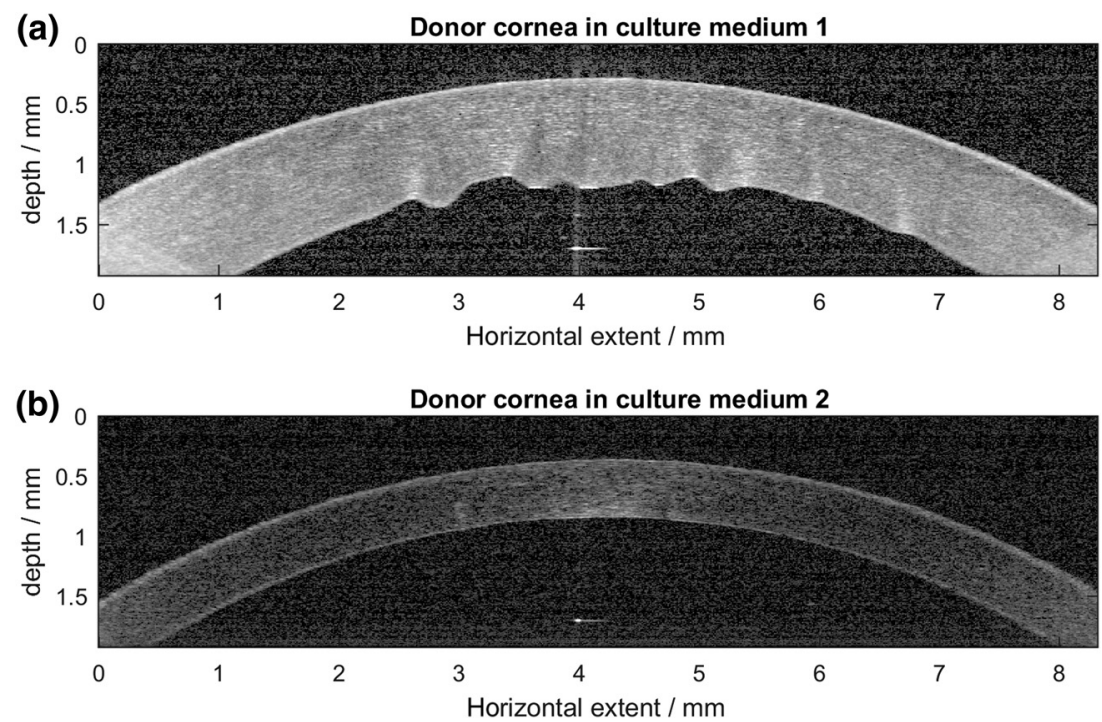

Fig. 1 OCT measurement image of corneal graft in culture medium 1 before reimmersement (a) and after reimmersement in culture medium 2 (b). The swelling of the cornea and the folding of the Descemet membrane can be seen on the posterior surface. After $\sim 48 \mathrm{~h}$ of deswelling in medium 2 , the same cornea shows no Descemet folds and a normal central thickness around $500 \mu \mathrm{m}$, but image contrast is reduced.

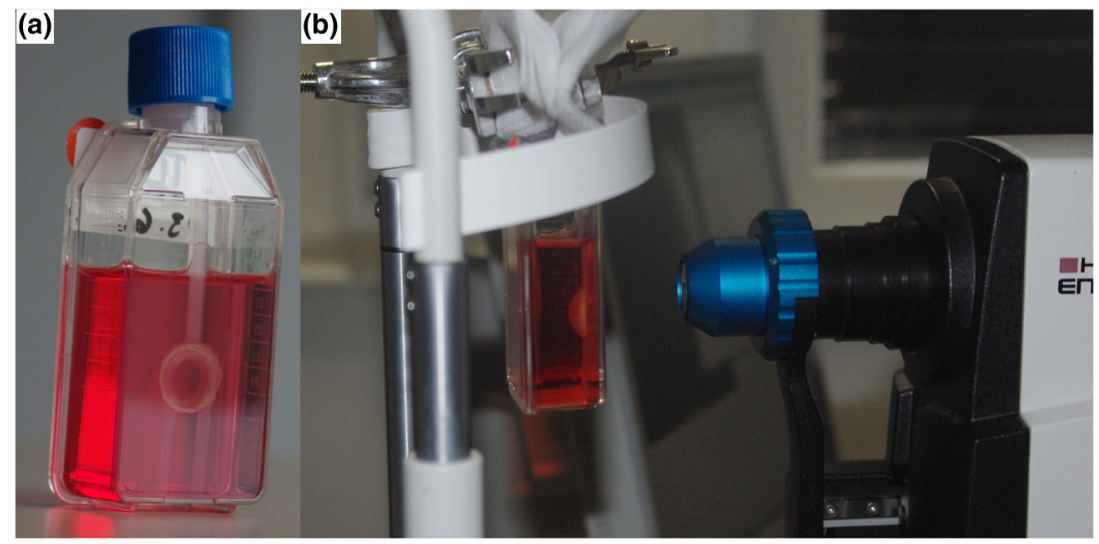

Fig. 2 Experimental setup. (a) The sterile tissue cultivation flask containing the donor cornea is mounted in front of the objective lens of the spectral-domain OCT system. (b) Donor cornea in sterile tissue flask cultivation.

culture flask are not equipped with antireflective coating, therefore, they reflect a perceptible portion of light stronger than the signal received from the corneal button. Techniques based on the reflection of placido disc mires are, therefore, unsuitable. In addition, particles from the corneal metabolism accumulate within the culture medium and lead to increased scattering. Reflections and scattering affect the image contrast. Furthermore, the optical resolution must be sufficient to measure density gradients within the stromal tissue and to derive the topography of the corneal anterior and posterior surfaces in detail while also providing data on the corneal stroma. Tomographic imaging based on slit projection principle and optical coherence tomography (OCT) provide a high spatial resolution. ${ }^{19,20}$ In addition, OCT-based imaging outperforms slit-projection when dealing with scattering particles and more intrinsic noise in the image data. ${ }^{21,22}$

Initial attempts for the detection of ablation patterns induced by previous keratorefractive surgery (e.g., LASIK, PRK, and radial keratotomy) were performed using the Orbscan (Bausch + Lomb, Rochester), a Placido and slit scanningbased topographer, on enucleated complete eye globes in air. ${ }^{23}$ Other investigations employed OCT-based methods because they enable the measurement of corneoscleral buttons under sterile conditions. ${ }^{24-27}$ LASIK interfaces could be detected within the reflection pattern of the stromal tissue. Beyond that, Lin et al. estimated anterior and posterior curvatures by means of a parabolic fit. Janunts et al. showed that keratoconus and extreme topographic irregularities could be identified by analyzing the thickness distribution of the cornea. ${ }^{28}$

To compute geometric parameters for classifying donor corneas, an appropriate surface model has to be chosen. The first approach was limited to spherical models with restriction to 1 degree of freedom (one radius of curvature $R$ ). With the use of conic sections, the model is extended by another parameter, the conic constant $\kappa$. It describes the contour of a conic section and thus considers the typical prolate ellipsoidal shape of the 
corneal surfaces. ${ }^{29}$ However, the normal human cornea is not rotationally symmetric. ${ }^{30}$ Therefore, the model needs to be expanded to a more general representation treating toroidal surfaces and two radii of curvature in orthogonal meridians. The biconic model is based on an explicit equation that allows four degrees of freedom, namely two radii of curvature and two conic constants. In this manner, both the cornea's asphericity and toricity are being addressed. ${ }^{31-33}$

The purpose of this study was to develop an OCT-based measurement procedure for automatic classification of corneal grafts that can be carried out during cultivation under sterile conditions.

\section{Material and Methods}

\subsection{Experimental Setup}

For the measurements, we used a clinical spectral-domain OCT system (SD-OCT) (Spectralis OCT, Heidelberg Engineering, Heidelberg, Germany) with a central wavelength of $\lambda=$ $870 \mathrm{~nm}$. To allow imaging of the cornea, it was equipped with an add-on objective lens (Anterior Segment Module, Heidelberg Engineering, Heidelberg, Germany). The rectangular polystyrene tissue cultivation flasks (Primaria $25 \mathrm{~cm}^{2}$ Canted-Neck Cell Culture Flask, Corning Inc., New York) containing the corneoscleral grafts [Fig. 2(a)] were mounted in front of the OCT system [Fig. 2(b)].

The corneal grafts were gathered from the LIONS cornea bank Saar-Lor-Lux, Trier/Westpfalz in Homburg/Saar and measured inside the sterile culture flask. The graft is fixated onto a polyethylene cornea holder with four brackets [Bausch + Lomb, Heidelberg, Germany; Fig. 2(a)]. The measurements were conducted at room temperature of $\sim 23^{\circ} \mathrm{C}$ and within 1 day after reimmersement because corneal metabolism particles obfuscate the culture medium, and the folding distorts the posterior surface topography. After measurement, the culture flasks were returned to the storing environment at $36^{\circ} \mathrm{C}$. The storage and transportation were done according to the German regulations for cornea banking. According to these the allowed temperature range in the second medium is between $10^{\circ} \mathrm{C}$ and $40^{\circ} \mathrm{C}^{10}$
The data were collected on the basis of a volume reconstruction of cross-sectional images through the posterior surface of the cornea (Fig. 3). The OCT was manually aligned to capture the central part of the cornea. The average penetration depth was $1.927 \mathrm{~mm}$ and the axial resolution $3.893 \mu \mathrm{m}$ across the corneal diameter of $8.3 \mathrm{~mm}$ [Fig. 4(a)]. The horizontal resolution was $10.821 \mu \mathrm{m}$. In total, the measured volume consisted of a vertical sequence of 81 cross-sectional images. With a distance of $69 \mu \mathrm{m}$ between each cross-section, the area had a vertical expanse of $5.52 \mathrm{~mm}$. After acquisition of the vertical array (scan 1), the scan window of the OCT was rotated by $90 \mathrm{deg}$ with the sample in place, and the acquisition was repeated (scan 2). The two volume scans were exported as video files (image stacks) and combined in one coordinate system.

\subsection{Data Analysis}

The data analysis was implemented in MATLAB (The MathWorks Inc., Massachusetts). The distinct steps of the procedure are shown in Fig. 5. At first, the region of interest (ROI) was applied to cutoff mirror artifacts in the periphery arising from the Fourier transform of the OCT fringe signal. ${ }^{34}$ The central $5.66 \mathrm{~mm}$ from the horizontal dimension of the corneal diameter ( $x$-axis) and the central $5.037 \mathrm{~mm}$ from the vertical dimension ( $y$-axis) were selected as ROI from the exported image files [Fig. 4(b)]. The cropped images had a resolution of $568 \times 496$ pixels with a scale of $10.821 \mu \mathrm{m} /$ pixel laterally and $3.893 \mu \mathrm{m} /$ pixel axially. Afterward, the cropped images were denoised, and the corneal volume was reconstructed as described in the following section. The two orthogonal measurements were placed in a common coordinate system [Fig. 4(a)] following an edge detection of the corneal surfaces. With the aid of a biconic surface fit, the two orthogonal radii of curvature and conic constants were determined.

\subsubsection{Denoising and edge detection}

Prior to the edge detection, the image data had to be refined from the remaining artifacts of the flask wall, reflections, and particles while maintaining the anterior and posterior corneal surfaces.
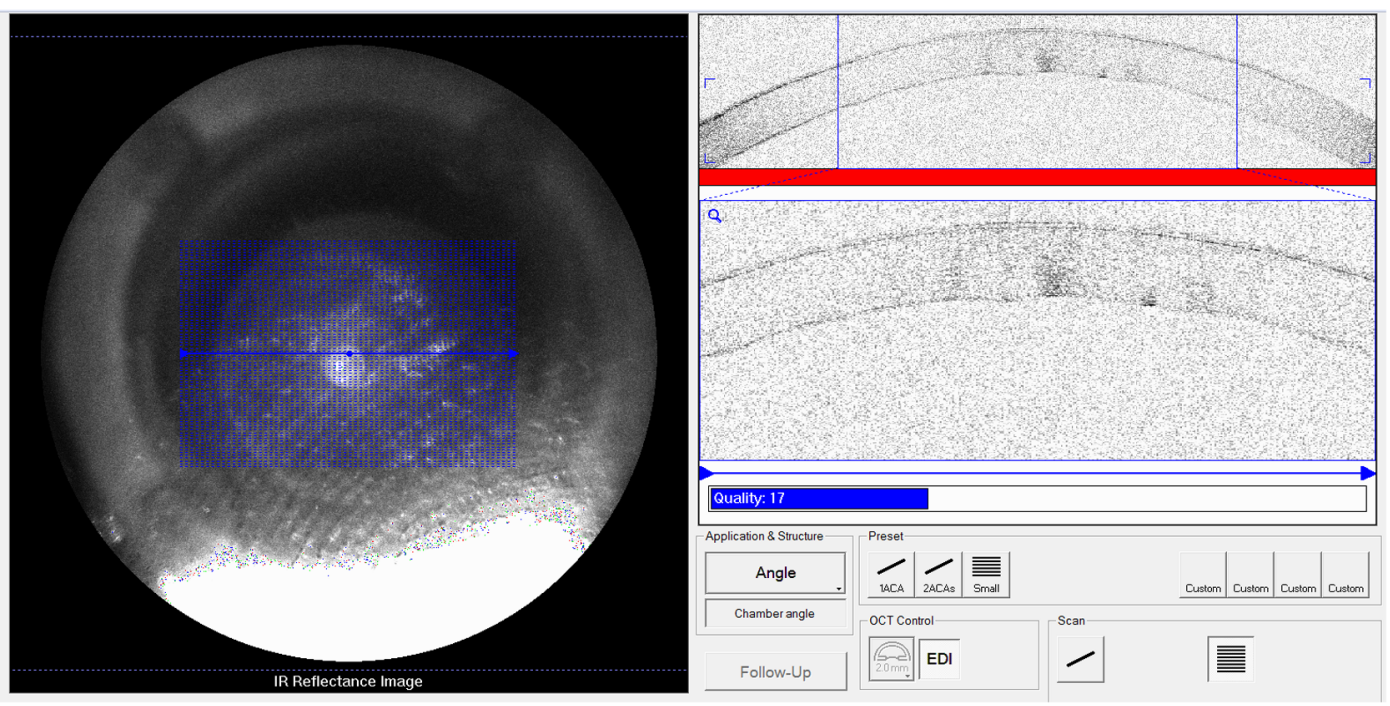

Fig. 3 OCT measurement software (Heidelberg Eye Explorer, Heidelberg Engineering, Heidelberg, Germany). The flask reflection is visible in the lower region of the left image. By tilting the culture flask, the reflected light portion is directed out of the scan area (blue grid in the IR reflectance image on the left side). 

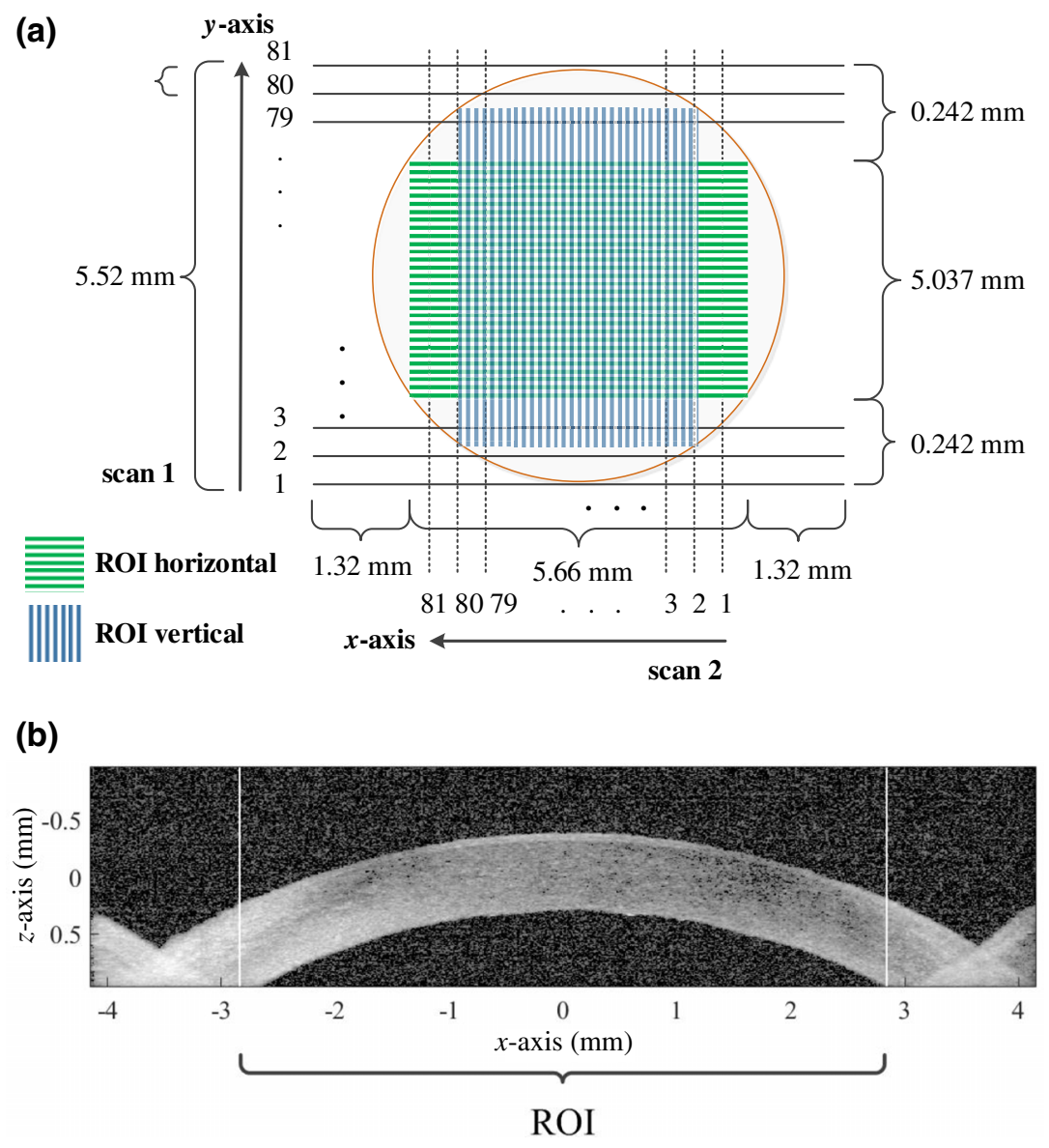

Fig. 4 Measuring scheme. (a) Scan area. The measured space was spanned of two overlapping measurements, each containing a $5.52 \mathrm{~mm} \times 8.3 \mathrm{~mm}$ array of 81 cross-sections (scan 1 and scan 2). The green and blue areas depict the resulting $(\mathrm{ROI}, 5.037 \mathrm{~mm} \times 5.66 \mathrm{~mm})$. (b) Sample image slice. Mirror artifacts are prevalent in the peripheral regions.

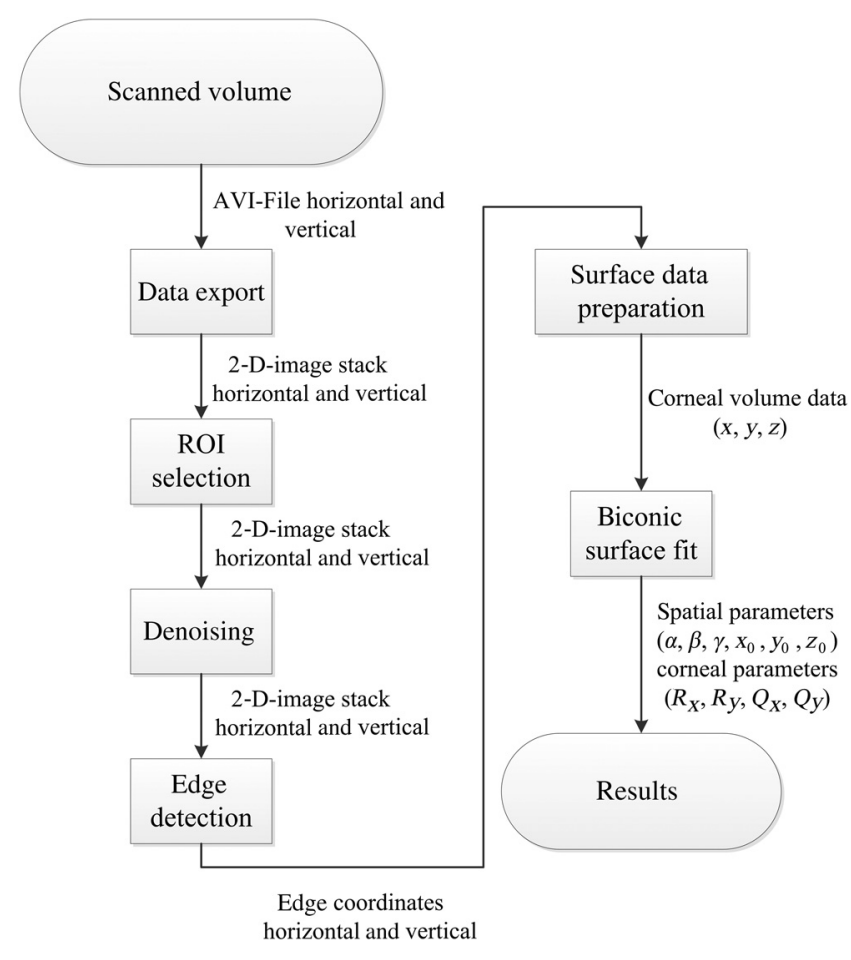

Fig. 5 Data analysis procedure.
For that reason, the background noise was eliminated with a $(5 \times 5)$ median filter. The number of particularly bright spots, such as reflections from the central corneal surfaces or mirrored artifacts from the back wall of the flask varied along the corneal diameter. The contrast range was adjusted by remapping the gray values of each image linearly to the interval $[0,1]$. Clustered background noise components and mirror artifacts were selectively removed by a morphological opening. The size of the structuring element was chosen to be large enough to remove salty noise while being as small as possible avoiding corneal edge distortion.

Variations in image brightness toward the periphery were corrected by adjusting the contrast in relation to the distance from the center. Remaining particles were removed by deleting small objects [Fig. 6(c)]. Missing parts of the corneal tissue due to the shadowing were filled up by a top-hat-transformation. This morphological operation performs another opening with a disk-shaped structuring element with a radius of 45 pixels. It was applied to the inverted image. As a result, the shadowings were opened as bright spots. The resulting image was subtracted from the original image. In this manner, the shadowed areas were closed. Finally, the edges were detected using the Canny method, ${ }^{35}$ starting with a low gradient threshold of 0.25 and including connected edge pixels up to a high gradient threshold of 0 . The resulting edges were assembled as surface data. 

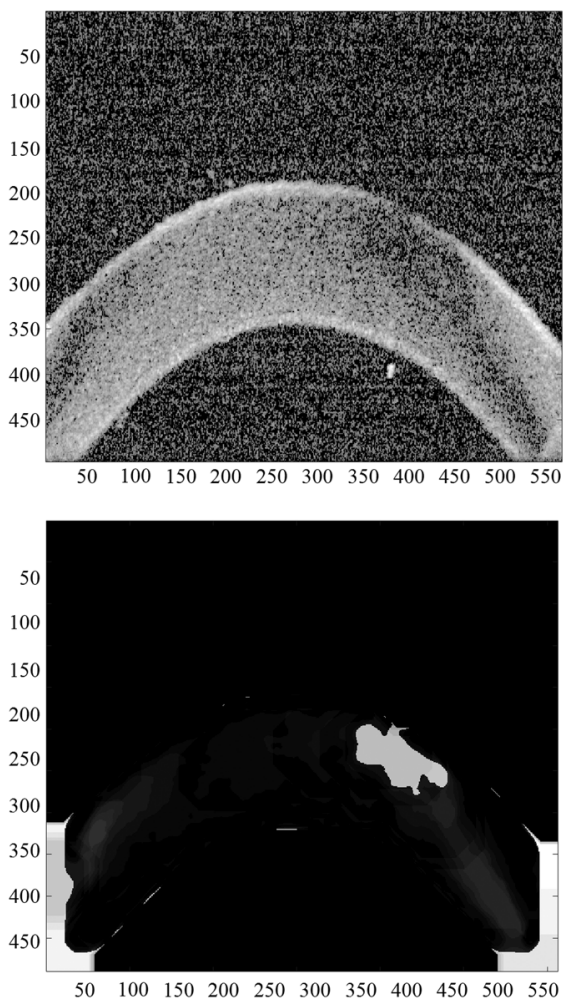
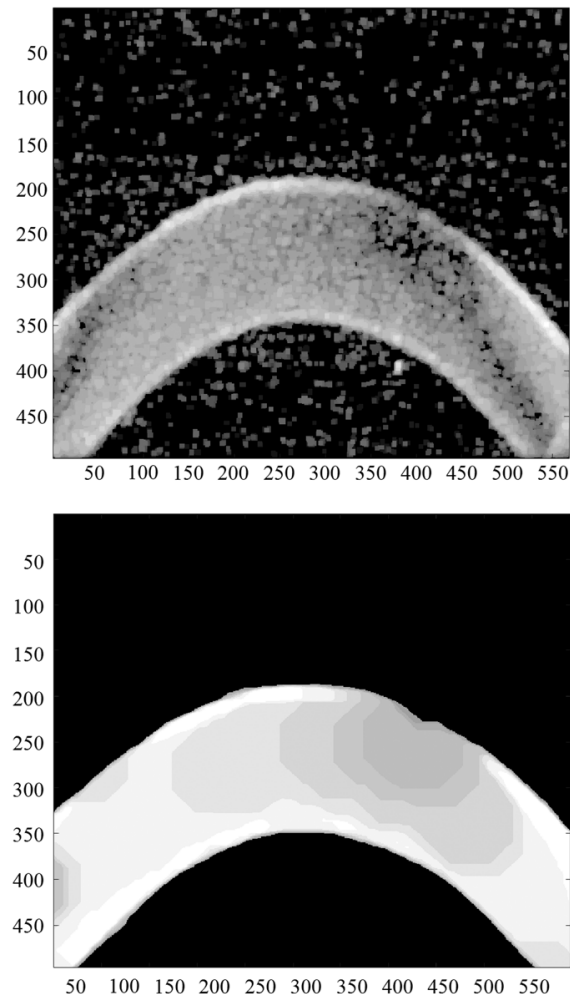
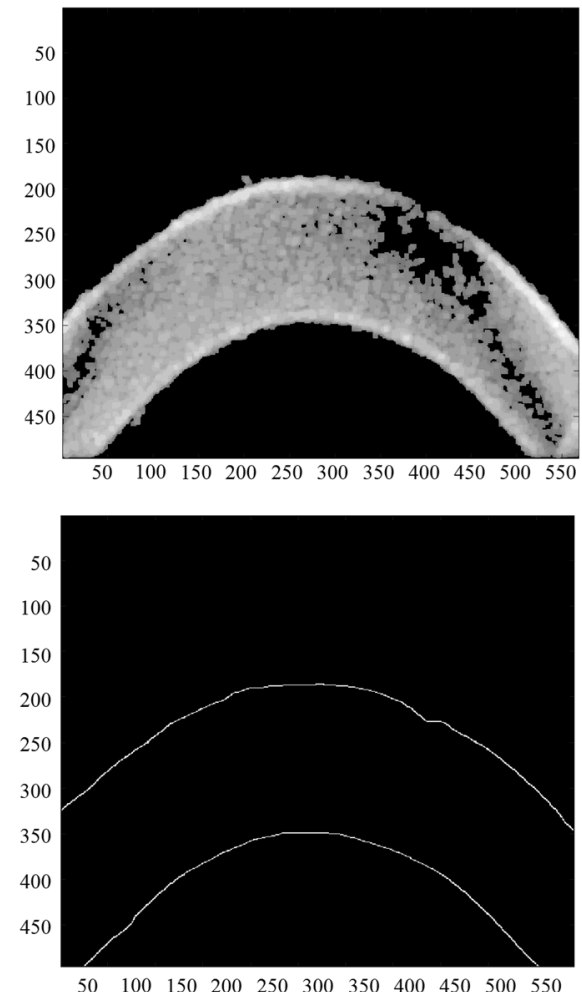

Fig. 6 Denoising routine, part 2. (a) Original image. (b) Morphologically opened image. (c) Image after removal of small particles. (d) Top-hat transform of the complementary image of (c). (e) image (c)-(d). (f) Detected edge. Gray values were scaled and do not reflect actual image gray values. Axes are in pixels.

\subsubsection{Biconic surface fit}

To determine two orthogonal radii of curvature $R_{x}$ and $R_{y}$ as well as the related conic constants $\kappa_{x}$ and $\kappa_{y}$, we expanded the well-known equation for the height data $z(x, y)$ of a biconic surface.

At first, the detected edges of the anterior and posterior surfaces from each scan were transformed into spatial coordinates. The penetration depth was defined as the $z$-axis, the horizontal expanse of each cross-section as the $x$-axis, and the vertical sequence of cross-sections as the $y$-axis. Then the corneal volume data of scan 1 and scan 2 were combined in one coordinate system $z(x, y)$ with $x=x_{\text {measurement } 1} \vee y_{\text {measurement2 }}$ and $y=y_{\text {measurement1 }} \vee x_{\text {measurement2 }}$.

To extract the required parameters, the biconic surface fit had to be independent from the spatial alignment of the corneoscleral button within the culture flask in relation to the measuring system.

For that reason, the spatial coordinates $x$ and $y$ were transformed to $x^{\prime}$ and $y^{\prime}$. The azimuthal rotation angle $\alpha$ of the orthogonal meridians around the $z$-axis was incorporated by multiplication of the coordinate vector $X=[x y]^{T}$ with a rotation matrix $R$. Translational shifts $x_{0}, y_{0}$ of the anticipatory apex $z=$ 0 from the origin of the coordinate system were addressed by subsequent multiplication with a translation matrix $\mathrm{T}$ and yielded the new coordinate vector $X_{\text {new }}=X R T$. The slant of the surface plane due to the varying fixation of the culture flask in relation to the OCT device, and the floating of the corneal graft was included by adding two tilted planes with the tilt angles $\beta$ and $\gamma$. Furthermore, the depth offset $z_{0}$ of the apex was subtracted from the resulting biconic equation. The final equation $z_{\text {model }}\left(x^{\prime}, y^{\prime}, \varepsilon\right)$ yields 10 degrees of freedom in terms of the corneal parameters $R_{x}, R_{y}, \kappa_{x}$, and $\kappa_{y}$ and the spatial parameters $x_{0}, y_{0}, z_{0}, \alpha, \beta$, and $\gamma$, merged in the parameter vector $\varepsilon=\left[R_{x}, R_{y}, \kappa_{x}, \kappa_{y} x_{0}, y_{0}, z_{0}, \alpha, \beta, \gamma\right]$ :

$$
\begin{aligned}
z_{\text {model }}\left(x^{\prime}, y^{\prime}, \varepsilon\right)= & \frac{\frac{x^{2}}{R_{x}}+\frac{y^{\prime 2}}{R_{y}}}{1+\sqrt{1-\frac{x^{\prime 2}}{R_{x}^{2}}\left(1+\kappa_{x}\right)-\frac{y^{\prime 2}}{R_{y}^{2}}\left(1+\kappa_{y}\right)}} \\
& -z_{0}+\tan (\beta) \cdot\left(x-x_{0}\right)+\tan (\gamma) \cdot\left(y-y_{0}\right) .
\end{aligned}
$$

The values of the corneal parameters for a given spatial setting were derived with an iterative nonlinear least squares fit with trust region algorithm. In the first step A of the fitting procedure (Fig. 7), the spatial parameters were estimated by fitting a rotationally symmetrical anterior and posterior surfaces with average radii of curvature $\left(R_{x}=R_{y}=7.8 \mathrm{~mm}\right.$ for the anterior corneal surface and $6.4 \mathrm{~mm}$ for the posterior corneal surface) and conic constants $\left(\kappa_{x}=\kappa_{y}=-0.2\right.$ anterior and -0.4 posterior $)^{36,37}$ to the measurement data. The initial spatial parameters of $\varepsilon_{0}$ were set to 0 . The root-mean-square error (RMSE) was calculated, and outliers were excluded from the data set.

In the second step $\mathrm{B}, z_{0}$ was assessed for the average cornea using the RMSE from step A as an initial parameter for $\varepsilon_{1}$. All the data points $z_{i}$ with residual $(i)>\operatorname{RMSE} \cdot 1.5$ were excluded. Afterward, the tilt angles $\beta$ and $\gamma$ were calculated for the average cornea with the $z_{0}$ value from step B. Data points $z_{i}$ with residual $(i)>\operatorname{RMSE} \cdot 1.2$ were discarded. The resulting data set $z_{i, \text { reduced }}\left(x_{\text {reduced }}, y_{\text {reduced }}\right)$ was used as the initial data set for steps $\mathrm{D}$ and $\mathrm{E}$ of the fitting procedure. 


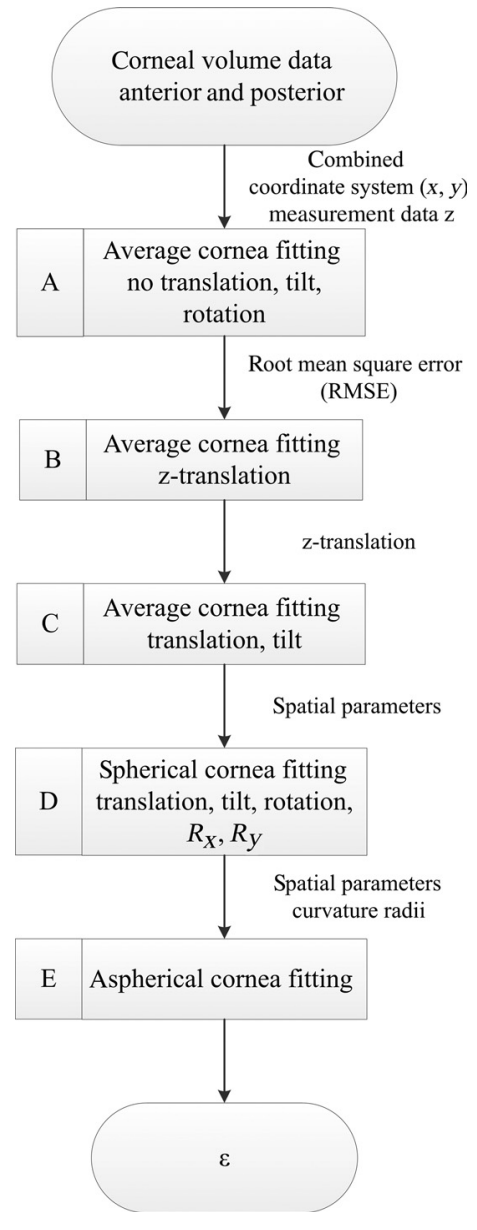

Fig. 7 Fitting procedure.

The previously probed values for $\beta, \gamma, x_{0}, y_{0}$, and $z_{0}$ were set as initial parameters for step $\mathrm{D}$, and the limits of their $95 \%$ confidence interval were set as lower and upper constraints. Then $R_{x}$ and $R_{y}$ as well as $\alpha$ were calculated iteratively until the fit converged, i.e., $\frac{\left|\varepsilon_{\text {iteration-1 }}^{n}-\varepsilon_{\text {iteration }}^{n}\right|}{\varepsilon_{\text {iteration-1 }}}<10^{-6}$ for each parameter $\varepsilon^{n}$ of $\varepsilon=\left(\varepsilon^{1}, \ldots, \varepsilon^{m}\right)$. Following each iteration, data points with $\operatorname{residual}(i)>Q_{0.0125}$ or $\operatorname{residual}(i)>Q_{0.9875}$ with $Q$ as the corresponding quantiles were ruled out. In the last fitting step, E, $\kappa_{x}$ and $\kappa_{y}$ were estimated while again using $\varepsilon_{\text {iteration-1 }}$ as initial parameters and the limits of their $95 \%$ confidence interval as constraints until the fit converged. Once more, the data points of the upper and lower $1.25 \%$ quantiles of the residual were excluded from the initial data set $z_{i, \text { reduced }}$ after each iteration.

\subsubsection{Additional parameters}

The orientation of the corneoscleral button on the corneal holder after excision and re-immersion is unknown. The flatter radius of curvature has been declared as $R_{\mathrm{f}}$, the steeper one as $R_{\mathrm{s}}$, and the associated conic constants as $\kappa_{\mathrm{f}}$ and $\kappa_{\mathrm{s}}$, respectively.

From the topographic data, the central corneal thickness (CCT) was determined by calculating the absolute distance between the anterior apex and the posterior surface

$\mathrm{CCT}=\left|z_{\text {anterior }}\left(x_{\text {apex }}^{\text {anterior }}, y_{\text {apex }}^{\text {anterior }}\right)-z_{\text {posterior }}\left(x_{\text {apex }}^{\text {anterior }}, y_{\text {apex }}^{\text {anterior }}\right)\right|$.

The refractive powers in the natural environment (interface air/cornea/aqueous humor) were calculated in both meridians $K$ and $K_{\mathrm{s}}$ for the anterior surface using Javal's keratometric approximation $K=\frac{n_{\mathrm{k}}-1}{R_{\text {anterior }}} \times 1000$ with $n_{\mathrm{k}}=1.3375$. The posterior surface power was calculated by means of the surface power equation $F=\frac{n_{\mathrm{a}}-n_{\mathrm{c}}}{R_{\text {posterior }}} \times 1000$ with $n_{\mathrm{a}}=1.336$ (aqueous, inner medium) and $n_{\mathrm{c}}=1.376$ (cornea, outer medium) to compare the outcome with clinical assessment of a physiological cornea using a Scheimpflug tomographer. The total refractive power or "true net power" (TNP) was computed by $\mathrm{TNP}=\frac{n_{\mathrm{c}}-1}{R_{\text {anterior }}} \times 1000+\frac{n_{\mathrm{a}}-n_{\mathrm{c}}}{R_{\text {posterior }}} \times 1000$ using the mean radius of curvature for each surface. Corneal astigmatism was calculated by the absolute difference of $K$ or $F$.

\section{Results}

To validate the measurement principle and fitting procedure, a rotationally symmetric model cornea made from polymethylmethacrylate (PMMA) was measured at first. The resulting parameter matched the theoretical specifications of the model lens with

Table 1 Radii of curvature $R$, conic constants $\kappa$, and keratometric power for the flat and steep meridians of the anterior and posterior corneal surfaces ( $n=23$ transplants). Data are given as mean \pm standard deviation. Anterior keratometric powers are given with respect to Javal's keratometric index.

\begin{tabular}{|c|c|c|c|c|}
\hline Parameter & Flat & Steep & Mean & Astigmatism \\
\hline \multicolumn{5}{|l|}{ Anterior surface } \\
\hline$R(\mathrm{~mm})$ & $8.03 \pm 0.38$ & $7.62 \pm 0.35$ & $7.83 \pm 0.12$ & $0.41 \pm 0.69$ \\
\hline$\kappa$ & $-0.286 \pm 0.241$ & $-0.252 \pm 0.163$ & $-0.269 \pm 0.174$ & - \\
\hline$K(D) n_{\mathrm{c}}=1.3375$ & $42.11 \pm 1.81$ & $44.38 \pm 2.47$ & $43.24 \pm 0.79$ & $2.27 \pm 4.04$ \\
\hline \multicolumn{5}{|l|}{ Posterior surface } \\
\hline$R(\mathrm{~mm})$ & $6.58 \pm 0.18$ & $6.26 \pm 0.27$ & $6.42 \pm 0.12$ & $0.33 \pm 0.39$ \\
\hline$\kappa$ & $-0.434 \pm 0.132$ & $0.418 \pm 0.145$ & $-0.426 \pm 0.132$ & - \\
\hline$F(D)$ & $-6.08 \pm 0.16$ & $-6.40 \pm 0.29$ & $-6.24 \pm 0.12$ & $0.32 \pm 0.40$ \\
\hline
\end{tabular}


Table 2 Corneal parameters of the suspected post-LASIK cornea (cornea 1), a cornea with extreme curvature radii (cornea 2), and the thickest cornea (cornea 3).

\begin{tabular}{|c|c|c|c|c|c|c|c|c|}
\hline \multirow[b]{2}{*}{ Parameter } & \multicolumn{2}{|c|}{$R_{\mathrm{f}}(\mathrm{mm})$} & \multicolumn{2}{|c|}{$R_{\mathrm{s}}(\mathrm{mm})$} & \multicolumn{2}{|c|}{$\kappa_{\mathrm{f}}$} & \multicolumn{2}{|c|}{$\kappa_{\mathrm{s}}$} \\
\hline & Anterior & Posterior & Anterior & Posterior & Anterior & Posterior & Anterior & Posterior \\
\hline Cornea 1 & 7.792 & 6.491 & 7.715 & 6.479 & -0.223 & -0.409 & -0.232 & -0.408 \\
\hline Cornea 2 & 9.214 & 7.121 & 6.099 & 5.568 & -1.074 & -0.306 & -0.844 & -0.041 \\
\hline Cornea 3 & 8.096 & 6.430 & 7.860 & 6.161 & -0.260 & -0.414 & -0.153 & -0.416 \\
\hline
\end{tabular}

Table 3 Refractive powers and CCT of the suspected post-LASIK cornea (cornea 1), a cornea with extreme curvature radii (cornea 2), and the thickest cornea (cornea 3). Anterior keratometric powers are given with respect to Javal's keratometric index.

\begin{tabular}{|c|c|c|c|c|}
\hline Parameters & & Cornea & Jornea & Cornea 3 \\
\hline$K_{\mathrm{f}}(D)$ & Anterior $n_{\mathrm{c}}=1.3375$ & 43.32 & 36.63 & 41.69 \\
\hline & Posterior & -6.16 & -5.62 & -6.22 \\
\hline$K_{\mathrm{s}}(D)$ & Anterior $n_{\mathrm{c}}=1.3375$ & 43.38 & 55.34 & 42.94 \\
\hline & Posterior & -6.17 & -7.18 & -6.49 \\
\hline Astigmatism $(D)$ & Anterior & 0.48 & 18.71 & 1.25 \\
\hline & Posterior & 0.02 & 1.57 & 0.27 \\
\hline ССТ $(\mu \mathrm{m})$ & & 540 & 675 & 844 \\
\hline$K_{\mathrm{m}}(D)$ & & 43.24 & 45.98 & 42.31 \\
\hline $\operatorname{TNP}(D)$ & & 42.32 & 42.80 & 40.77 \\
\hline
\end{tabular}

a relative error of $\Delta R_{x}=0.4 \%, \Delta R_{y}=1.2 \%, \Delta \kappa_{x}=0.32 \%$, $\Delta \kappa_{y}=0.08 \%$, and $\Delta \mathrm{CCT}=2.2 \%$. Then 23 corneal grafts from 18 donors (11 left and 12 right eyes) were screened. One graft was anamnestically suspected to have undergone LASIK surgery. The measurements were conducted 1 day after the transfer to the deswelling culture medium, and the transplantation was scheduled within the next 4 days. The corneal radii of curvature, $\kappa$ values, and the estimated keratometric power values and astigmatism are shown in Table 1 . The CCT was $616 \pm 100 \mu \mathrm{m}$ (455 to $844 \mu \mathrm{m})$.

Three extreme cases are presented in Table 2. Cornea 1 represents a historically suspected post-LASIK cornea. Cornea 2 represents the highest amount of astigmatism, whereas cornea 3 represents the thickest graft. The corresponding refractive powers and CCT are displayed in Table 3.

\section{Discussion}

\subsection{Corneal Parameters}

In this study, the geometrical properties of corneal grafts were assessed by means of an OCT-based screening method. The donor corneas were maintained in their sterile cultivation flasks within the eye bank's storage procedure. Using a biconic surface fit, two orthogonal radii of curvature and the corresponding conic constants $\kappa$ were elicited from the anterior and posterior corneal surfaces, respectively. Refractive power and astigmatism were calculated in a way that allows direct comparison with in vivo measured corneas. The corneal thickness, particularly at the anterior apex (CCT), was derived from the determined corneal volume model. The median radii of curvature of $R_{\mathrm{f} \text {,anterior }}=$ $7.890 \mathrm{~mm}, \quad R_{\mathrm{s}, \text { anterior }}=7.705 \mathrm{~mm}, \quad R_{\mathrm{f} \text {,posterior }}=6.538 \mathrm{~mm}$, and $R_{\mathrm{s} \text {,posterior }}=6.354 \mathrm{~mm}$ correspond to average horizontal and vertical corneal curvatures found in previous studies in vivo. ${ }^{33,37,38}$ The mean CCT of $616 \mu \mathrm{m}$ is slightly higher than the average value found in normal human corneas $(534 \mu \mathrm{m}) .{ }^{39}$ This effect can be attributed to the cultivation process of the graft as the culture medium increases stromal hydration. ${ }^{40}$ We conducted our measurements within 1 day after the grafts had been reimmersed in the deswelling medium, and in some cases, this time span might have been insufficient. The deswelling of organ-cultured corneal tissue was investigated by Schnitzler et al. who found that the condition of the endothelium might have a significant effect on the deswelling aside from the osmotic effect of the culture medium. ${ }^{27}$ Thus, measuring corneal thickness before reimmersement and at a standardized time after reimmersement might be one indicative factor for endothelium activity.

Schnitzler et al. reported a minimal corneal thickness after $24 \mathrm{~h}$ of deswelling. ${ }^{27}$ We chose a time span of 1 day as a good compromise between the time for deswelling and residual OCT image contrast.

One cornea was anamnestically reported to have undergone refractive surgery (cornea 1 ). The type of refractive surgery, however, was unknown. However, the corneal parameters, refractive power, and the CCT $(539.9 \mu \mathrm{m})$ did not differ significantly from the median values. The visual inspection of cornea 1 did not reveal any signs for refractive surgery. Previous studies found that LASIK interfaces can be seen in the short term in the stromal reflectivity data as bright spots. For longer periods of time, the interface intensity fades and manifests in a lower internal reflectivity. ${ }^{26}$ Lin et al. found the LASIK corneas to have flatter anterior curvature radii, which could not be detected in cornea 1 of this study.

Cornea 2 with the flattest anterior curvature radius showed a large discrepancy between the two curvature radii $R_{\mathrm{f}}=$ $9.214 \mathrm{~mm}$ and $R_{\mathrm{S}}=6.099 \mu \mathrm{m}$ and is, therefore, causing the extreme outlier within the astigmatism values $(20.84 \mathrm{D})$. The corresponding conic constants of the anterior surface also show extreme values $\left(\kappa_{\mathrm{f}}=-1.074\right.$ and $\left.\kappa_{\mathrm{s}}=-0.844\right)$. Although the posterior surface is within the average range, this cornea exhibits the highest anterior refractive power $\left(D_{\mathrm{f}}=61.65 \mathrm{dpt}\right)$.

With cornea 3, all corneal parameters except for the CCT $(844.28 \mu \mathrm{m})$ were close to the median values. However, the ratios of the anterior to posterior curvature radius $(0.794$ and 
0.784 for the flatter and the steeper meridian, respectively) are lower than the average ratio (0.82).

\subsection{Fitting Procedure}

Due to the manual mounting of the cultivation flask in front of the objective of the OCT, the exact position of the corneal graft was unknown and not reproducible. By involving rotation, tilt angles, and translation parameters, the automatic fitting procedure was made independent of the alignment of the donor cornea to the corneal holder and its positioning within the culture flask.

Because of the rotationally nonsymmetrical toroidal surface of the cornea, a general biconic representation was elected as a mathematical model for the nonlinear least squares fitting procedure. In contrast to the ellipsoidal model, the individual parameters are independent from each other. ${ }^{41}$ The denoising routine yielded a smooth edge that eliminates particles and reflections and preserves the corneal surface. During the fit, we used two different refinement criteria to exclude outliers in the residual data. The first restricted the data to 1.5.RMSE, the second one to 1.2.RMSE. These criteria were arbitrarily chosen as a compromise between quality of the fit and convergence of the fit while still considering a sufficient amount of data. On average, $29 \pm 20$ iterations were necessary until the fit converged (minimum: 6, maximum: 95). The average RMSE was $15.13 \mu \mathrm{m} \pm 13.30 \mu \mathrm{m}$ (minimum: $3.72 \mu \mathrm{m}$ and maximum: $69.32 \mu \mathrm{m}$ ), and the highest values occurred when the number of iterations culminated.

Calculating the biconic surface with the average anterior radii of curvature $\left(R_{\mathrm{f}}=8.030 \mathrm{~mm}\right.$ and $\left.R_{\mathrm{s}}=7.624 \mathrm{~mm}\right)$ and conic constants $\left(\kappa_{\mathrm{f}}=-0.286\right.$ and $\left.\kappa_{\mathrm{s}}=-0.252\right)$, a difference of $\Delta \kappa=$ -0.1 manifests in the peripheral regions of the measurement area $(-2.83 \mathrm{~mm}$ and $2.83 \mathrm{~mm})$ in an elevation difference of $1.7 \mu \mathrm{m}$. Considering the depth resolution of $3.893 \mu \mathrm{m}$ and the mean RMSE of the fitting procedure, this calculation of $R$ and $\kappa$ is rather inaccurate compared with clinical measurement methods. The average human corneal diameter (horizontal limbus-to-limbus distance) is $11 \mathrm{~mm} \cdot{ }^{36}$ Screening a larger, circularshaped area would significantly improve the calculation of the conic constant. Preliminarily estimating the unknown location of the corneal graft in relation to the OCT device by fitting an average cornea reduces the number of fitting parameters. Especially in the peripheral regions, a difference in the elevation data can almost equally be assigned to either different corneal parameters or varying tilt and rotation angles. This may lead to an inconclusive fitting result. To eliminate this drawback, the spatial parameters have been estimated beforehand in steps A-D of the fitting procedure (Fig. 6). The accuracy of this anticipatory approach has been verified with the PMMA model lens. Tilt, translation (especially relative to the optical axis of the OCT's objective lens), and asymmetry of the cornea will affect the quality of the measurement data and the surfacefitting procedure, as they might induce significant refraction or displacement of the OCT beam. In the fitting procedure tilt, translation and corneal asymmetry will lead to errors in the biconic fit due to fitting the data to different data points. Furthermore, keratometric values will be compromised as well. Therefore, some care should be taken for a properly placed specimen during the image acquisition procedure. This is, in fact, one of the limitations in the study, the upright measurement procedure using a clinical OCT and the fact that the cornea might most probably not be in its natural geometric state, will affect our results (compare cornea 2). Alternative fixation techniques for corneal grafts are available that might help to reduce this problem. In addition, a marker on the corneal graft may assist proper alignment to improve the results. However, regulatory decrees do not allow an easy change of the fixation procedure. Another limitation arises from the OCT technique. One should take care of correcting the refraction of the rays at the first refractive surface of the cornea, which is especially important when measuring the cornea in air due to the high difference in the refractive indices between cornea and air. Exact measurement of geometrical parameters also requires correction of refraction at the interface between culture medium and cornea. However, we assumed that this effect would be rather small due to the small difference between the refractive indices of these media. The resulting error in thickness measurement due to neglecting the optical path differences between the surrounding medium and the corneal tissue was estimated to be $<3 \%$.

The use of Zernike polynomials for the reconstruction of corneal surfaces either as a global approach or as an additional decomposition of the residual error from a basic surface fit has been widely discussed in the literature. ${ }^{41-43}$ While optical aberrations can be sufficiently modeled by Zernike decomposition and might be beneficial in the detection of keratoconus, ${ }^{44,45}$ the coefficients yield no direct information about geometrical properties such as curvature or asphericity. Moreover, the number of coefficients necessary to minimize the RMSE when representing abnormal corneas increases rapidly.

\subsection{Optical Coherence Tomography Technique}

In comparison with other measurement methods like x-ray or magnetic resonance techniques, OCT offers the best spatial resolution and does not harm the examined tissue. ${ }^{20}$ Unlike confocal microscopy, it is independent from the aperture of the system and provides a higher penetration depth. For that reason, OCT has become a widespread measurement method in ophthalmology, ${ }^{46}$ and it has previously been applied for screening corneal grafts aside from imaging the cornea in vivo. ${ }^{22,24,26,28,40,47}$ Considering the necessity of a sterile environment for the graft, the use of a contact-free measurement technique is obligatory. Because of the reflective behavior of the flask walls, other noncontact, high-resolution measurement methods, such as slit-projection or Placido topography would require the exposure of the tissue to air. Ultrasound techniques necessitate the application of an immersion medium, which is less comfortable.

The imaging modality of the OCT device exerts an influence on the signal-to-noise ratio and duration of the measurement. SDOCT imaging offers several advantages over time-domain OCT (TD-OCT) devices. The Spectralis OCT is a Fourier-domain OCT (FD-OCT) and has a broadband light source, which simultaneously collects the measurement signal of multiple wavelengths. Therefore, it works much faster than the sequential image acquisition of TD-OCT devices. Although FD-OCT devices show difficulties in eliminating speckle and electron noise, several studies have shown that they offer a higher sensitivity and better spatial resolution, especially for the transparent tissue of the eye, but not in highly scattering tissue. ${ }^{48-50}$

\section{Conclusion}

Using SD-OCT, eye banks' donor corneas could be observed under sterile conditions in their culture flasks to detect major 
curvature abnormalities. Using a biconic surface fit with 10 degrees of freedom, the curvature radii and conic constants could be estimated for the anterior and posterior corneal surfaces.

To further elaborate the screening method, the accuracy can be refined by expanding the scan range. The development of a custom-built, portable screening device ${ }^{47,49,51,52}$ would facilitate the integration of the screening procedure into the eye banks' cultivation procedure. Moreover, the donor-host matching could be improved by labeling the meridians of the corneoscleral button and extraction of dioptric values before excision. The influence of the excision, cultivation, and transplantation procedure on the imaging quality of the corneal graft could be assessed and compared with the clinical outcome of the transplantation. In this way, the transplants could be selected specifically and aligned during surgery to compensate preexisting host astigmatism. The intensity gradient of the stromal tissue and the thickness profile should be taken into account as well to identify previous LASIK and (preclinical) keratoconus on the corneal grafts.

\section{Disclosures}

The authors have no financial interest to declare in this manuscript.

\section{Acknowledgments}

We gratefully acknowledge the financial support provided by the Dr. Rolf M. Schwiete Foundation. We would like to thank Mr. Herbert Junger from Heidelberg Engineering for the support with the anterior segment module and the Lions Cornea Bank Saar/Trier/Westpfalz for providing the corneal grafts.

\section{References}

1. E. Zirm, "Eine erfolgreiche totale Keratoplastik," Graefes Arch. Ophthalmol. 64, 580-593 (1906).

2. P. Gain et al., "Global survey of corneal transplantation and eye banking," JAMA Ophthalmol. 134(2), 167-173 (2016).

3. H. Reinshagen et al., "Aktivitaten der Sektion Gewebetransplantation und Biotechnologie der Deutschen Ophthalmologischen Gesellschaft. 4. Leistungsbericht 2013," Ophthalmologe 112(1), 70-72 (2015).

4. H. Reinshagen et al., "Aktivitaten der Sektion Gewebetransplantation und Biotechnologie der Deutschen Ophthalmologischen Gesellschaft: Leistungsbericht 2014," Klin Monbl Augenheilkd 233(11), 1266-1269 (2016).

5. B. Seitz, A. Langenbucher, and G. O. H. Naumann, "Die perforierende Keratoplastik," Ophthalmologe 102(12), 1128-1139 (2005).

6. B. Seitz et al., "Hornhauttransplantation: Zu geringe Spendenbereitschaft," Dtsch. Ärztebl. 101(49), A3326-A3330 (2004).

7. L. Pels, "Organ culture: the method of choice for preservation of human donor corneas," Br. J. Ophthalmol. 81(7), 523-525 (1997).

8. A. S. Neubauer et al., "Sterile structural imaging of donor cornea by optical coherence tomography," Cornea 21(5), 490-494 (2002).

9. J. Schroeter et al., "Augenhornhaut—Banken und klinische Anwendung," Transfus. Med. Hemother. 35, 431-437 (2008).

10. Bundesärztekammer-Wissenschaftlicher Beirat, "Richtlinie zur Gewinnung von Spenderhornhäuten und zum Führen einer Augenhornhautbank," Dtsch. Ärztebl. 111(31-32), A-1386-C-1138 (2014).

11. Eye Bank Association of America Medical Advisory Board, Medical Standards, Washington D.C. (2015).

12. D. Glasser, "Donor eligibility/suitability," Int. J. Eye Bank. 3(2), 1-4 (2015).
13. J.-F. Laliberté et al., "Topography-based screening for previous laser in situ keratomileusis to correct myopia and hyperopia," Cornea 24(2), 167-177 (2005).

14. M. A. Terry and P. J. Ousley, "New screening methods for donor eye-bank eyes," Cornea 18(4), 430-436 (1999).

15. S. J. Kang et al., "Donor corneas misidentified with prior laser in situ keratomileusis," Cornea 29(6), 670-673 (2010).

16. S. C. Belmont et al., "Keratoconus in a donor cornea," J. Refract. Corneal Surg. 10(6), 658 (1994).

17. A. Michaeli-Cohen et al., "Two cases of a penetrating keratoplasty with tissue from a donor who had undergone LASIK surgery," Cornea 21(1), 111-113 (2002).

18. R. A. Fargione and P. Channa, "Cornea donors who have had prior refractive surgery: data from the Eye Bank Association of America," Curr. Opin. Ophthalmol. 27(4), 323-326 (2016).

19. R. C. Drews, "Depth of field in slit lamp photography. An optical solution using the Scheimpflug principle," Ophthalmologica 148, 143-150 (1964).

20. A. F. Fercher, "Optical coherence tomography-development, principles, applications," Z. Med. Phys. 20(4), 251-276 (2010).

21. T. Eppig et al., "Contact free in-vivo imaging of cornea and anterior chamber of the human eye-a qualitative comparison of imaging techniques," Klin. Monatsbl. Augenheilkd. 228(12), 1052-1059 (2011).

22. K. Moutsouris et al., "Optical coherence tomography, Scheimpflug imaging, and slit-lamp biomicroscopy in the early detection of graft detachment after Descemet membrane endothelial keratoplasty," Cornea 30(12), 1369-1375 (2011).

23. S. Hick et al., "Topographic screening of donor eyes for previous refractive surgery," J. Cataract Refract. Surg. 32(2), 309-317 (2006).

24. S. G. Priglinger et al., "Optical coherence tomography for the detection of laser in situ keratomileusis in donor corneas," Cornea 22(1), 46-50 (2003).

25. A. H. Wolf et al., "Detection of laser in situ keratomileusis in a postmortem eye using optical coherence tomography," J. Cataract Refract. Surg. 30(2), 491-495 (2004).

26. R. C. Lin et al., "Screening for previous refractive surgery in eye bank corneas by using optical coherence tomography," Cornea 26(5), 594599 (2007).

27. A. C. Schnitzler et al., "Role of the endothelial layer in the deswelling process of organ-cultured human corneas before transplantation," Cornea 35(9), 1216-1221 (2016).

28. E. Janunts, A. Langenbucher, and B. Seitz, "In vitro corneal tomography of donor cornea using anterior segment OCT," Cornea 35(5), 647-653 (2016).

29. A. Calossi, "Corneal asphericity and spherical aberration," J. Refract. Surg. 23(5), 505-514 (2007).

30. P. M. Kiely, G. Smith, and L. G. Carney, "The mean shape of the human cornea," Opt. Acta 29(8), 1027-1040 (1982).

31. A. Langenbucher, A. Viestenz, and B. Seitz, "Conoidal fitting of corneal topography height data after excimer laser penetrating keratoplasty," J. Refract. Surg. 18(1), 63-71 (2002).

32. H. Burek and W. A. Douthwaite, "Mathematical models of the general corneal surface," Ophthalmic Physiol. Opt. 13(1), 68-72 (1993).

33. E. Janunts, M. Kannengießer, and A. Langenbucher, "Parametric fitting of corneal height data to a biconic surface," Z. Med. Phys. 25(1), 25-35 (2015).

34. J. Ho et al., "Clinical assessment of mirror artifacts in spectral-domain optical coherence tomography," Invest. Ophthalmol. Vis. Sci. 51(7), 3714-3720 (2010).

35. J. Canny, "A computational approach to edge detection," IEEE Trans. Pattern Anal. Mach. Intel. PAMI-8(6), 679-698 (1986).

36. K. P. Mashige, "A review of corneal diameter, curvature and thickness values and influencing factors," Afr. Vis. Eye Health 72(4), 185-194 (2013).

37. M. Dubbelman, V. A. D. P. Sicam, and G. L. van der Heijde, "The shape of the anterior and posterior surface of the aging human cornea," Vis. Res. 46(6-7), 993-1001 (2006).

38. P. M. Kiely, G. Smith, and L. G. Carney, "Meridional variations of corneal shape," Am. J. Optom. Physiol. Opt. 61(10), 619-626 (1984).

39. M. J. Doughty and M. L. Zaman, "Human corneal thickness and its impact on intraocular pressure measures: a review and meta-analysis approach," Surv. Ophthalmol. 44(5), 367-408 (2000). 
40. Y. Wu et al., "Noninvasive optical coherence tomography monitoring of structure and hydration changes of human corneas in different preservation media," J. Biomed. Opt. 16(2), 026015 (2011).

41. R. Navarro, L. González, and J. L. Hernández, "Optics of the average normal cornea from general and canonical representations of its surface topography," J. Opt. Soc. Am. A 23(2), 219-232 (2006).

42. M. K. Smolek and S. D. Klyce, "Goodness-of-prediction of Zernike polynomial fitting to corneal surfaces," J. Cataract Refract. Surg. 31(12), 2350-2355 (2005).

43. Z. Zhu et al., "Iteratively re-weighted bi-cubic spline representation of corneal topography and its comparison to the standard methods," Z. Med. Phys. 20(4), 287-298 (2010).

44. J. Bühren, T. Schäffeler, and T. Kohnen, "Validation of metrics for the detection of subclinical keratoconus in a new patient collective," J. Cataract Refract. Surg. 40(2), 259-268 (2014).

45. R. Shetty et al., "A novel Zernike application to differentiate between three-dimensional corneal thickness of normal corneas and corneas with keratoconus," Am. J. Ophthalmol. 160(3), 453-462 (2015).

46. C. A. Puliafito et al., Optical Coherence Tomography of Ocular Diseases, SLACK Inc., Thorofare, New Jersey (1996).

47. P. E. Napoli et al., "Post-mortem corneal thickness measurements with a portable optical coherence tomography system: a reliability study," Sci. Rep. 6, 30428 (2016).

48. M. A. Choma et al., "Sensitivity advantage of swept source and Fourier domain optical coherence tomography," Opt. Express 11(18), 21832189 (2003).

49. R. Leitgeb, C. Hitzenberger, and A. Fercher, "Performance of Fourier domain vs. time domain optical coherence tomography," Opt. Express 11(8), 889-894 (2003).

50. B. Liu and M. E. Brezinski, "Theoretical and practical considerations on detection performance of time domain, Fourier domain, and swept source optical coherence tomography," J. Biomed. Opt. 12(4), 044007 (2007).

51. D. Nankivil et al., "Handheld, rapidly switchable, anterior/posterior segment swept source optical coherence tomography probe," Biomed. Opt. Express 6(11), 4516-4528 (2015).

52. H. Akil et al., "Identification of anterior chamber angle parameters with a portable SD-OCT device compared to a non-portable SDOCT," Int. Ophthalmol. (2016).
Angela Damian is a scientist at the Institute of Experimental Ophthalmology, Saarland University in Homburg/Saar. She received her bachelor's degree in electrical engineering (energy and automation) from Frankfurt University of Applied Sciences in 2013 and her master's degree in biomedical engineering from the University of Applied Sciences in Saarbruecken in 2015. Her research interests include the development of automatic optical measurement technology, diagnostic systems for the human eye, electrical systems, and neural engineering.

Berthold Seitz is a full professor and the chairman of the Department of Ophthalmology, Saarland University Medical Center in Homburg/ Saar. He completed his residency in ophthalmology in 1993 and he is a renowned expert in corneal diseases, corneal surgery, eye banking, and corneal transplantation. He was appointed as the head of the University Eye Hospital at the Saarland Medical Center in 2006. His current research interests include keratoconus and new therapeutic options for corneal pathologies.

Achim Langenbucher is a full professor and the head of the Institute of Experimental Ophthalmology, Saarland University in Homburg/ Saar. He received his diploma degree in electrical engineering and his doctoral degree from the University of Erlangen-Nuremberg in 1995. He was appointed as an associate professor for medical optics at the Institute of Medical Physics in Erlangen in 2005. He is the chairman of Experimental Ophthalmology, Saarland University since 2009. His research interests include laser therapy in ophthalmology and individualized ophthalmology.

Timo Eppig is an adjunct professor at the Institute of Experimental Ophthalmology, Saarland University in Homburg/Saar. He received his diploma degree in optical technology and image processing from the Darmstadt University of Applied Sciences in 2005 and his doctoral degree from the University Erlangen-Nuremberg in 2009. $\mathrm{He}$ works as a research associate at the Institute of Experimental Ophthalmology at Saarland University since 2009. His research interests include individualized ophthalmology, automated diagnostics, intraocular lenses, and optical design. 